\title{
Why $2+2=5$ looks so wrong: On the odd-even rule in sum verification
}

\author{
LESTER E. KRUEGER and ERNEST W. HALLFORD \\ Ohio State University, Columbus, Ohio
}

\begin{abstract}
The odd-even status of a sum depends on the odd-even status of its addends. A sum must be odd if an odd number of its addends are odd; else it must be even. A proposed sum that violates the required odd-even status of the sum-that is, deviates from the correct sum, whether odd or even, by an odd value (e.g., splits of $\pm 1, \pm 3, \pm 5$ )-can be rejected immediately as false. Subjects in the present study did indeed use the odd-even rule in sum verification, because they were as fast and accurate in rejecting a split of \pm 1 as one of \pm 2 , and a split of \pm 3 as one of \pm 4 , even though a larger split generally is easier to reject (symbolic distance effect), and splits of \pm 3 and \pm 4 were rejected faster and more accurately than those of \pm 1 and \pm 2 . Performance on separate odd-even tasks indicated that the odd-even properties of numbers and sums are readily available for use by adults, and that persons who do well on such tasks are especially likely to use the odd-even rule in sum verification.
\end{abstract}

Why is $2+2=5$ used so often to illustrate an obvious error in addition? Why not use $2+2=6$, which contains an even larger error? After all, the amount of time required to decide which of two digits is larger decreases as the magnitude of the difference between the two digits increases (Banks, Fujii, \& Kayra-Stuart, 1976; Moyer \& Landauer, 1967; Restle, 1970), and Ashcraft and Battaglia (1978) found that the same "symbolic distance effect" holds in sum verification. With ties excluded, false response time (RT) was much higher if the discrepancy or split between the proposed sum and the correct sum was a "reasonable wrong" of \pm 1 or \pm 2 $(1,144 \mathrm{msec})$ rather than an "unreasonable wrong" of \pm 5 or $\pm 6(1,044 \mathrm{msec})$, and that true RT was lowest of all $(1,016 \mathrm{msec})$.

An apparent exception to the symbolic distance effect is the failure to find a decrease in false RT when the split is increased from \pm 1 (e.g., $2+2=5$ ) to \pm 2 (e.g., $2+2=6$ ). Parkman and Groen (1971), using a within-subjects experimental design, found that false judgments were slightly faster in the \pm 1 case $(777 \mathrm{msec})$ than the \pm 2 case $(788 \mathrm{msec})$, and fastest of all on true trials $(707 \mathrm{msec})$. In a pilot study, Hallford (1982, Experiment 1) varied split between subjects, using equations that had a correct sum of 7,8 , or 9 and that contained as many as five nonzero addends. He, too, found

This study was supported in part by National Institute of Mental Health Grant MH32295. Experiment 1 was conducted as part of a master's thesis by Ernest W. Hallford (1982) under the supervision of Lester E. Krueger. The authors are grateful to Ronald G. Shapiro for assistance in data collection and analysis, and to Mark H. Ashcraft, Mark H. Chignell, and Steven W. Keele for helpful comments on an earlier version of this report. Requests for reprints should be sent to Lester E. Krueger, Human Performance Center, Ohio State University, 404-B West 17th Avenue, Columbus, Ohio 43210.

-Steven W. Keele served as Action Editor for this manuscript. that false RT was slightly faster $(\mathrm{F}<1)$ in the \pm 1 case $(2,502 \mathrm{msec})$ than in the \pm 2 case $(2,504 \mathrm{msec})$, with true judgments being faster than either by about $125 \mathrm{msec}$. Also, errors did not differ between the \pm 1 and \pm 2 groups $(F<1)$. However, neither study tested larger splits, and it may be that some other aspect of the method than the use of \pm 1 and \pm 2 splits, such as the relatively insensitive between-subjects design used in the latter case, eliminated the symbolic distance effect. The present study used a within-subjects experimental design and a wider range of splits $( \pm 1, \pm 2, \pm 3, \pm 4)$ to verify that the symbolic distance effect holds between other splits even when it is not found between \pm 1 and \pm 2 .

The symbolic distance effect may actually hold between \pm 1 and \pm 2 , but simply be counteracted by a factor favoring a fast decision in the \pm 1 case. With $2+2$ as addends, a proposed answer of 5 may look as wrong as one of 6 because it violates the odd-even rule in sum verification. If both addends are even, as with $2+2$, or both are odd, then the correct sum must be even, which thus excludes 5 , but not 6 . The odd-even rule in sum verification may be stated more formally as follows: If one and only one of the two addends is odd, then the correct sum must be odd; else it must be even. The rule might be implemented in various ways. One way is simply to tally the number of odd terms and to respond "false" immediately if one or all three terms (two addends, one proposed sum) are odd, but to complete the regular addition operation if none or two are odd.

The rule may be generalized as follows for more than two addends: If an odd number of addends are odd, then the correct sum must be odd; else it must be even. The odd-even rule is violated whenever the proposed sum deviates from the correct sum, whether odd or even, by an odd value (e.g., splits of $\pm 1, \pm 3, \pm 5, \pm 7$, etc.). Whenever the proposed sum deviates from the correct sum by 
an even value, on the other hand, it retains the same odd-even status as the correct sum, whether odd or even.

The present study tested splits of $\pm 1, \pm 2, \pm 3$, and \pm 4 . If both the odd-even status and symbolic distance affect performance, then RT and errors should decrease with increased split (symbolic distance effect), but not in a smooth fashion, owing to dips in RT and errors on splits of \pm 1 and \pm 3 . Use of the odd-even rule should counteract the symbolic distance effect between \pm 1 and \pm 2 and between \pm 3 and \pm 4 , but should reinforce it between \pm 2 and \pm 3 . Therefore, it was predicted that the biggest improvement in performance with increased split would occur between \pm 2 and \pm 3 . An alternative explanation of such a finding is that splits of \pm 1 and \pm 2 are "reasonably wrong," whereas splits of \pm 3 and \pm 4 are "unreasonably wrong" (Ashcraft \& Battaglia, 1978). However, there is no reason to expect the breakpoint between reasonably and unreasonably wrong to be located consistently just between a split of \pm 2 and a split of \pm 3 .

The odd-even rule has considerable aid to offer in sum verification. At one stroke, it halves the number of possible correct answers. In particular, it can eliminate the most troublesome potential error of all, namely, those false sums that deviate from the correct sum by \pm 1 . Miller, Perlmutter, and Keating (1984) used a production task and found that $31 \%$ of the addition errors made by their adult subjects deviated from the correct sum by \pm 1 . Thus, many errors could be avoided simply by using the odd-even rule to check the answer, and the rule may well be worth teaching explicitly in elementary school.

The present study investigated whether adults spontaneously use the odd-even rule when shown test equations in a true-false verification task. Unlike the production task, which requires subjects to supply the answer, the verification task provides subjects with a proposed sum, which must be classified as true or false. The verification task may seem rather artificial, but some evidence indicates that it provides a more valid assessment of the processes used in arithmetic (Ashcraft, Fierman, \& Hamann, 1980). Furthermore, it provides a ready means for measuring the ease with which particular false sums are rejected.

\section{EXPERIMENT 1}

Experiment 1 used splits of $+1,+2,+3$, and +4 to test the odd-even rule. Only positive discrepancies were used, both for simplicity and because on negative splits subjects may rely on partial answers, making an immediate "no" or "false" response as soon as the accumulating count exceeds the proposed sum (cf. Stazyk, Ashcraft, \& Hamann, 1982). Each equation contained two addends, whose correct sum ranged from 10 to 17 . This allowed the use of every possible combination of addends for each particular correct sum (see Table 1). The range 10 to 17 was used so that each correct sum would contain two digits, and so that we could test the effect of the size of the correct sum and minimum (smaller) addend on adult RT. With children, Groen and Parkman (1972) found that the size of the minimum addend had a large effect on RT, and they proposed a counting model: A counter is set to the value of the maximum (larger) addend (which is assumed to take a constant amount of time and to determine the intercept of the RT function), and then is incremented the number of times indicated by the value of the minimum addend (which determines the slope of the RT function). However, with adults, the size of the correct sum predicted RT nearly as well as did the size of the minimum addend, and the slope for minimum addend was only $5 \%$ as large as the slope for children (Parkman \& Groen, 1971). They proposed that adults rely largely on direct access and only rarely on counting (5\%) in making their decisions. Size of correct sum or correct sum squared has sometimes predicted adult RT better than has size of minimum addend (Ashcraft, Fierman, \& Hamann, 1980; Ashcraft \& Stazyk, 1981, Experiment 2; Gonzalez \& Kolers, 1982), thus giving further credence to the contention (Ashcraft \& Hamann, 1981) that mental addition for adults is more a matter of memory retrieval than of computation.

For half of the subjects in Experiment 1, a plus sign preceded each number, whether addend or proposed sum. For the other half, a minus sign appeared in place of the plus sign. The intent was to determine whether negative numbers are more difficult to deal with than positive numbers. There was no difference on either RT or errors ( $F \cong 1$ in both cases), and this variable will not be discussed further.

\section{Method}

Subjects. Twenty-four Ohio State University undergraduates participated in order to receive credit in an introductory psychology course. Data were excluded on four additional subjects whose errors exceeded $8 \%$. All subjects had at least $20 / 30$ vision (corrected) as tested with a Snellen chart.

Apparatus. Arabic numerals were presented at a $60-\mathrm{Hz}$ refresh rate and at $32 \mathrm{~cd} / \mathrm{m}^{2}$ intensity on a greenish-tint, fastdecay P31 phosphor (decay to $1 \%$ intensity at $.25 \mathrm{msec}$ after display offset) by an Imlac PDS-4 graphics computer, which measured RT to an accuracy of $1 \mathrm{msec}$. The numerals were software generated using short line vectors and were presented as thin luminous lines on a dark screen. Each subject sat alone in a dark room, with the head held fast in a chinrest located $70 \mathrm{~cm}$ from the display screen.

Stimulus materials. Each character was $.43 \mathrm{~cm}$ high $\times .29 \mathrm{~cm}$ wide, with $.14 \mathrm{~cm}$ between adjacent characters. An equal sign separated the two addends on the left from the proposed sum on the right, and each of the three numbers was preceded by a plus sign for half of the subjects and by a minus sign for the other half. Each equal sign and plus or minus sign was preceded by a blank space $(.43 \mathrm{~cm}$ wide). The equation had a total width of $5.47 \mathrm{~cm}(4.47 \mathrm{deg})$. The correct sums on regular trials ranged from 10 to 17 , and all 60 possible addend pairs that produce sums in this range were used (see Table 1). All addends were presented as two-digit numbers; addends below 10 were preceded by zero.

Eighteen practice trials preceded the main set of 498 trials (18 distractors, 480 regular trials), for a total of 516 trials. Half of the 480 regular trials showed true sums, and the other half were divided equally among false sums with splits of $+1,+2$, 
Table 1

Experiment 1: Sixty Sums and Addend Pairs Used to Generate All Trials

\begin{tabular}{lllllllllll}
\hline CS & \multicolumn{10}{c}{ Addend Pairs } \\
\hline 10 & $0+10$ & $1+9$ & $2+8$ & $3+7$ & $4+6$ & $5+5$ & & & \\
11 & $0+11$ & $1+10$ & $2+9$ & $3+8$ & $4+7$ & $5+6$ & & & \\
12 & $0+12$ & $1+11$ & $2+10$ & $3+9$ & $4+8$ & $5+7$ & $6+6$ & & \\
13 & $0+13$ & $1+12$ & $2+11$ & $3+10$ & $4+9$ & $5+8$ & $6+7$ & & \\
14 & $0+14$ & $1+13$ & $2+12$ & $3+11$ & $4+10$ & $5+9$ & $6+8$ & $7+7$ & \\
15 & $0+15$ & $1+14$ & $2+13$ & $3+12$ & $4+11$ & $5+10$ & $6+9$ & $7+8$ & \\
16 & $0+16$ & $1+15$ & $2+14$ & $3+13$ & $4+12$ & $5+11$ & $6+10$ & $7+9$ & $8+8$ \\
17 & $0+17$ & $1+16$ & $2+15$ & $3+14$ & $4+13$ & $5+12$ & $6+11$ & $7+10$ & $8+9$ \\
\hline
\end{tabular}

Note - CS $=$ correct sum

+3 , or +4 . Each of the 60 addend pairs shown in Table 1 was presented eight times: four times on true trials, and once for each of the four splits on false trials. Eight of the 18 distractors showed true sums of $18,19,20$, or 21 , so as to eliminate any upper boundary effect near 17 , and 10 showed a false sum of 10,11 , or 12 (e.g., $+06+02=+10$ ), so as to eliminate any lower boundary effect near 10 . The two addends in each equation were randomly ordered.

Procedure. On each trial, the test equation was shown until the subject responded. During the $1.2 \mathrm{sec}$ interval between trials, no feedback was given on the accuracy of the response.

The subjects were asked to respond as quickly as possible, but to try to avoid making errors. Half of the subjects pressed a left-hand button for "true" and a right-hand button for "false," and the other half had the reverse hand assignment.

Mean RT was based only on correct responses, and trials were discarded if the RT was below $200 \mathrm{msec}$ or over $15 \mathrm{sec}$.

\section{Results}

As Figure 1 shows, true (vs. false) trials were significantly faster, 1,205 msec versus $1,367 \mathrm{msec}[\mathrm{F}(1,22)=$ $51.67, \mathrm{p}<.001$ ], and slightly, but insignificantly, less accurate, $4.41 \%$ versus $4.10 \%(F<1)$. Size of correct sum had a significant effect on $\operatorname{RT}[F(7,154)=7.02$, $\mathrm{p}<.001$ ] , reflecting mainly an abnormally low RT for a correct sum of 10 (vs. 11 to 17), but no effect on errors $(\mathrm{F}<1)$. Trial type (true, false) interacted significantly with size of correct sum on RT $[F(7,154)=3.11$, $\mathrm{p}<.01]$. The speed advantage for true trials was much stronger when the correct sum was 10 than when it was any other value (see Figure 1).

Figure 2 plots the RT data for each of the 60 addend pairs by the size of the minimum (smaller) addend. Separate analyses at each size of correct sum indicated that RT increased significantly in each case as the size of the minimum addend increased from zero to its maximum possible value ( $p<.001$ for all eight sums, 10 to 17). As shown by the filled circles connected by dashed lines in Figure 2, however, there generally was no increase at all in RT when one of the addends was 10 and the other was nonzero, or when the two addends were identical to one another (i.e., ties). Excluding the latter two types of trials, the best-fitting linear regression function had an intercept of $1,116 \mathrm{msec}$ and a slope of $66.12 \mathrm{msec}$ on size of the minimum addend $\left(\mathbf{R}^{2}=\right.$ $0.7390)$. [When two other deviant pairs $(4+6 ; 8+9)$, whose RTs also were especially fast, were excluded too, size of minimum addend predicted RT somewhat better $\left(R^{2}=0.8441\right)$, with an intercept of $1,103 \mathrm{msec}$ and a slope of $74.33 \mathrm{msec}$.] Analyses of errors showed no effect of size of minimum addend.

Trial type (not shown in Figure 2) affected mainly the intercept. For true trials alone, the intercept was $1,046 \mathrm{msec}$, the slope was $62.68 \mathrm{msec}$, and $\mathbf{R}^{2}=0.6060$. For false trials alone, the intercept was $1,185 \mathrm{msec}$, the slope was $69.56 \mathrm{msec}$, and $\mathrm{R}^{2}=0.7340$. When the size of the maximum (larger) addend was analyzed, the intercept was $1,669 \mathrm{msec}$, the slope was $-37.72 \mathrm{msec}$, and $R^{2}=0.3107$. The negative slope is artifactual and reflects the confounding between the size of maximum and minimum addends. Given the fixed range of sums, the largest maximum addends (e.g., 16, 17) could occur only with the smallest minimum addends (e.g., 0,1 ), which, of course, produced the fastest responses. If the maximum and minimum addends were equally important, then RT should have increased steadily as the size of the correct sum, which gives equal weight to the two addends, increased. However, size of correct sum predicted $\mathrm{RT}$ very poorly $\left(\mathrm{R}^{2}=0.0150\right)$, with an intercept of $1,189 \mathrm{msec}$ and a slope of $8.93 \mathrm{msec}$. As stated above, correct sum had a significant effect on RT, but only because equations having a correct sum of 10 tended to produce faster judgments (see Figure 1).

On false trials analyzed separately, split had a significant effect on RT $[F(3,66)=9.85, p<.001]$ and errors $[\mathrm{F}(3,66)=5.87, \mathrm{p}<.01]$, with both generally decreas.

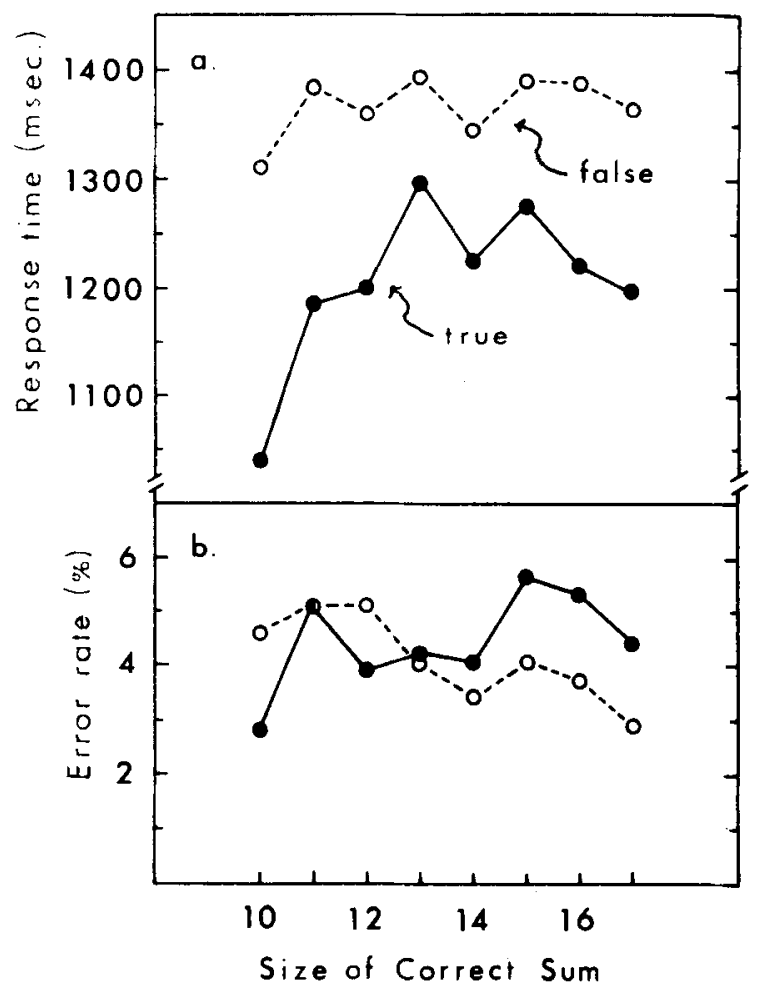

Figure 1. Experiment 1: Mean response time (in milliseconds) and percentage of error rate by trial type and size of correct sum. 


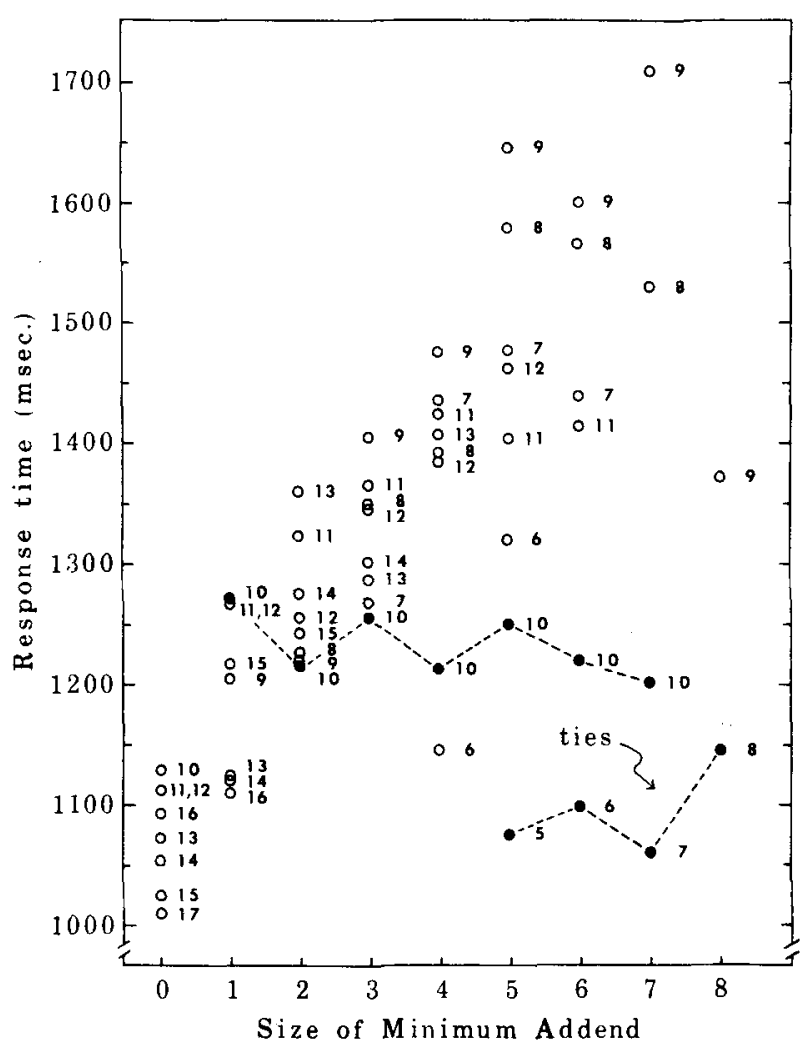

Figure 2. Experiment 1: Mean response time (in milliseconds) by size of minimum addend (horizontal axis) and maximum addend (parameter values next to each point).

ing as the split increased (i.e., symbolic distance effect). As Figure 3 shows, though, the general reduction in RT and errors was accompanied by downward dips at splits of +1 and +3 , just as predicted by the oddeven rule. Orthogonal components of the split effect were computed in order to assess the strength of these two tendencies. The linear component, reflecting the linear portion of the symbolic distance effect, was significant on $\operatorname{RT}[F(1,66)=27.08, p<.001]$ and errors $[F(1,66)$ $=9.89, \mathrm{p}<.01]$. The quadratic component, reflecting curvature or nonlinearity in the symbolic distance effect, was not significant on $\mathrm{RT}(\mathrm{F}<1)$ or errors $(\mathrm{F}<1)$. The cubic component, reflecting the zigzag pattern predicted by the odd-even rule, was significant on errors $[F(1,66)=7.01, p<.01]$, but not on $\operatorname{RT}[F(1,66)=$ 2.07]. Pairwise comparisons of adjacent splits showed no effect for a split of +1 versus +2 on $\operatorname{RT}[F(1,22)=$ 1.50] or errors $[F(1,22)=2.67]$, or for +3 versus +4 on $\mathrm{RT}(\mathrm{F}<1)$ or errors $(\mathrm{F}<1)$, but a strong effect on both RT $[F(1,22)=10.74, p<.001]$ and errors $[F(1,22)$ $=8.12, \mathrm{p}<.001]$ between +2 and +3 (see Figure 3 ).

\section{Discussion}

Experiment 1, in accord with Hallford (1982, Experiment 1) and Parkman and Groen (1971), found no difference between a split of +1 and a split of +2 . There also was no difference between splits of +3 and +4 . These are not due simply to an insensitive procedure, because the general decreases in RT and errors with increased split (linear component) were significant, and both speed and accuracy improved highly significantly when the split increased from +2 to +3 . The zigzag pattern (cubic component), which was significant on errors, is consistent with the use of the odd-even rule in sum verification.

The data indicate further that a violation of the oddeven rule has about the same effect as a unit increase in symbolic distance. Each reduced RT by about $30 \mathrm{msec}$ and errors by about $1 \%$. The symbolic distance factor was somewhat stronger than the odd-even factor on RT, which decreased when the split increased from +1 to +2 or from +3 to +4 , but not on errors, which increased when the split increased from +1 to +2 (see Figure 3).

When questioned after the session, nine subjects said they sometimes noticed that the proposed sum was even, whereas the true sum was odd, or vice versa. Five of the nine said they made use of this information, two noting that when the sum should be odd then even must be wrong and another two saying simply that this information led to "quicker" or "easier" responses. In addi-

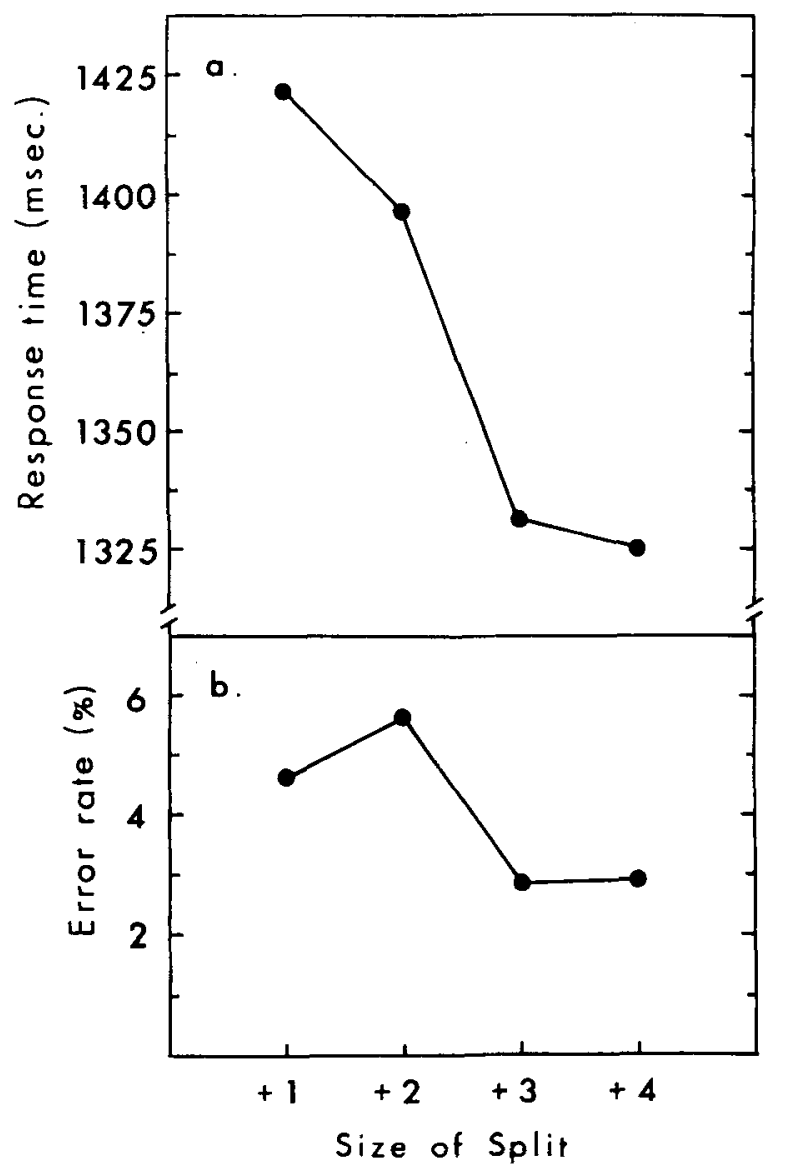

Figure 3. Experiment 1: Mean response time (in milliseconds) and percentage of error rate by size of split (false equations only). 
tion, a pilot subject reported using the odd-even rule on even addends, for which "odd was not possible as an answer." However, the fact that most subjects were unaware of having used some form of the odd-even rule, even though their data indicate that they did so, suggests that they usually used the rule in an automatic fashion, that is, as a form of tacit knowledge or skill.

The subjects in Experiment 1 used several other cues besides violations of the odd-even rule to bypass the normal addition operation. Ties had a low, nearly constant RT (see Figure 2), as several other investigators also have found (e.g., Aiken \& Williams, 1973; Ashcraft \& Battaglia, 1978; Ashcraft \& Stazyk, 1981; Parkman \& Groen, 1971). This suggests that ties are processed differently than nonidentical pairs of addends, whether by special direct access or by a doubling rule (Ashcraft \& Hamann, 1981). Adding 10 to a nonzero digit also produced a low, nearly constant RT (see Figure 2), although not quite as low as on ties, perhaps because a double-digit addend (i.e., 10) was involved, whereas the ties used here all had only addends below 10 . The subjects had only to replace the 0 in 10 with the other addend on these pairs. Addend pairs whose correct sum was 10 also tended to be fast, although not as consistently so as the ties and the pairs having an addend of 10 . Aiken and Williams (1973) also found that pairs summing to 10 were easier to process, which they attributed to the greater familiarity of these pairs.

For the remaining trials, which permitted no bypassing of normal addition, mean RT was generally well predicted by the size of the minimum (smaller) addend, and by that alone. Except for the abnormally low RT with a sum of 10 , there was no effect of size of correct sum, contrary to previous studies (Ashcraft, Fierman, \& Hamann, 1980; Ashcraft \& Stazyk, 1981, Experiment 2; Gonzalez \& Kolers, 1982). This may be because only larger problems (correct sums of 10 to 17) were used here, or because frequency increased as the correct sum increased. In the present set of 60 pairs, as Table 1 shows, six pairs each had a correct sum of 10 or 11 , but nine pairs each had a sum of 16 or 17. Various details, particularly the computed sum, may be retained in memory after solving an addition problem (Russo \& Wisher, 1976), and the extra exposure here to the larger sums may simply have compensated for less prior experience with the larger numbers (cf. Aiken \& Williams, 1973). In contrast, in previous studies, problems having larger sums were usually underrepresented rather than overrepresented, so the real effect of correct sum may have been exaggerated in an upward rather than a downward direction in those cases. Typically, subjects were given the 100 basic facts of addition obtained by crossing the 10 single digits $(0-9)$ with each other. This produces a positive correlation between the size of the minimum and maximum addends (see Parkman, 1972, Table 2), in contrast to the negative correlation in Experiment 1 (see Table 1). It also produces very few sums in the upper region near 18, but many sums in the intermediate region near 10 . This may have prompted subjects to expect very few large sum answers, thus slowing the RT on large-sum problems to such an extent that the correct sum squared sometimes predicted RT better than did the correct sum (e.g., Ashcraft \& Battaglia, 1978).

Much higher values on the intercept $(1,116 \mathrm{msec})$ and the slope by size of the minimum addend $(66 \mathrm{msec})$ were obtained here than were obtained on nontie trials by Parkman and Groen (1971) (intercept of about 700 msec; slope of about $20 \mathrm{msec}$ ) and by Ashcraft and Battaglia (1978) (intercept of about $950 \mathrm{msec}$; slope of about $35 \mathrm{msec}$ ). Given that children have a slope of about $400 \mathrm{msec}$, Groen and Parkman (1972) took their 20. msec slope value as evidence that adults count up to the minimum addend from the maximum addend only $5 \%$ of the time. If so, then the present adults counted up about $15 \%$ of the time when generally larger sums were tested. Counting up to a small minimum addend might often have been easier than relying on memory retrieval, since the equations for the larger sums may generally be less well established in memory. It is possible, however, that the subjects never counted up at all, and that the 66-msec slope represents merely an increase in the difficulty of memory retrieval as a function of the size of minimum addend (Ashcraft \& Stazyk, 1981; Winkelman \& Schmidt, 1974).

\section{EXPERIMENT 2}

Experiment 2 tested directly whether the odd-even properties of numbers can be accessed and dealt with sufficiently rapidly to aid the sum verification decision. Unfortunately, in addition to being unfamiliar to most people, tasks dealing directly with odd-even properties have various limitations. Therefore, two tasks, each having different limitations, were used in the hope of obtaining converging evidence on the approximate speed and accuracy with which oddness and evenness can be processed.

In the first odd-even task, the oddness tally task, the subjects had to determine how many of the three terms in each equation (i.e., two addends, one proposed sum) were odd. If subjects can readily count the number of odd (or even) terms in a three-term equation, that would indicate a capability to check the numbers and then respond "false" immediately if one or all three were odd. We did not have subjects make a binary decision between correct equations ( 0,2 odd numbers) and incorrect ones (1,3 odd numbers), because pilot tests had indicated that considerable practice would be required to master this manner of grouping the four types of trials. Instead, the subjects simply indicated how many odd terms $(0,1,2,3)$ they found. The equations varied on different blocks of trials as to their falseness, in order to determine whether numerical properties would be attended to on this task, even though they were irrelevant. 
The oddness tally task has a limitation in that subjects must choose among four responses. Furthermore, people may not apply the odd-even rule by counting the number of odd (or even) terms. Therefore, a second oddeven task, the odd-even choice task, also was used. Two addends were followed by an equal sign and then a question mark. The subjects judged whether the correct sum of the two addends had to be odd or even. They were told to respond "even" if both numbers were even or both were odd, but "odd" if one number was odd and the other was even. The limitation on this task was that subjects simply might add the two terms and decide whether the sum obtained that way was odd or even. Subjects might be particularly prone to add if it is quite difficult to code the odd-even properties of the two numbers. To check on this possibility, the subjects were asked after the session how frequently they had relied on addition in this task.

The addition task also was used in Experiment 2, primarily to obtain a performance baseline for the two odd-even tasks (oddness tally, odd-even choice), and secondarily to test whether performance would be the same for splits of \pm 1 and \pm 2 or \pm 3 and \pm 4 , but not for splits of \pm 2 and \pm 3 . To extend the generality of the results of Experiment 1, negative $(-1,-2,-3,-4)$ as well as positive $(+1,+2,+3,+4)$ splits were used. Furthermore, since the odd-even rule is valid for subtraction as well as addition, a subtraction (difference verification) task also was used.

In order not to bias performance on addition and subtraction, those two tasks were presented before the two odd-even tasks. All subjects had the following order of tasks: addition, subtraction, oddness tally, and oddeven choice. Because of the many changes in task, and the need to instruct and monitor the subjects more fully on the odd-even tasks, the subjects were tested with sheets of equations. Since performance was timed for an entire sheet (20 trials), not for individual trials, RT could not be distinguished for true and false trials on the arithmetic tasks, which may attenuate the effect of split. However, the present paper-and-pencil tests were deemed quite adequate to achieve the main goal, namely, measuring relative performance on the arithmetic tasks and the odd-even tasks.

\section{Method}

Subjects. Twenty-four Ohio State University undergraduates participated in order to receive credit in an introductory psychology course.

Stimulus materials. A separate set of 28 sheets (4 practice, 24 regular) was prepared for each subject. Six (practice sheet) or 20 (regular sheet) equations were double-spaced on a $10.32 \mathrm{~cm}$ wide $\times 21.59 \mathrm{~cm}$ high sheet. The sheet was detached from a larger computer printout sheet that contained the correct answers. The equations began $1.40 \mathrm{~cm}$ from the top of the sheet and extended for $3.49 \mathrm{~cm}$ ( 6 equations) or $12.38 \mathrm{~cm}$ (20 equations) down the sheet. Each equation was $3.02 \mathrm{~cm}$ wide, and to its right were blanks for the answer. All numbers were presented as double digits, for example, 09, 00, 13 .

The same basic set of 498 equations was used that had been used in Experiment 1. Eighteen of the 498 equations, half of which were true and half were false, were selected randomly for use in the practice sheets, and the remaining 480 were used only in regular sheets. The 18 practice equations were ordered randomly and divided into 3 sets of 6 each, with each set being used equally often across subjects as the practice set for the addition, subtraction, and oddness tally tasks. One set was reused for the odd-even choice task. The 480 regular equations were ordered randomly and divided into 24 sets of 20 each. Each set was used once as each of the 24 regular sheets ( 8 addition, 8 subtraction, 4 oddness tally, 4 oddeven choice) across the 24 subjects.

In the addition and subtraction tasks, the size of split varied on the practice sheet, but was fixed at one value $(-1,-2,-3$, $-4,+1,+2,+3$, or +4 ) on each of the eight regular sheets. To form a subtraction equation, the first and third terms in the corresponding addition equation exchanged places, and the plus sign was changed to a minus sign. Thus, $01+16=17$ in addition became $17-16=01$ in subtraction.

In the oddness tally task, two of the four regular sheets contained about half true equations, whereas the other two contained only false equations. On two sheets, the split varied over a small range, that is, 0 versus \pm 1 or \pm 1 versus \pm 2 , whereas on the other two it varied over a larger range, that is, 0 versus \pm 3 or \pm 1 versus \pm 4 . In the odd-even choice task, a question mark, ?, replaced the proposed sum, for example, $11+03$ $=$ ?, and the four regular sheets did not differ on any variable.

Procedure. The four tasks always were presented in the following order: (1) addition, (2) subtraction, (3) oddness tally, (4) odd-even choice. On each task, the subjects first received detailed instructions, then saw a demonstration sheet containing five equations with the correct answers checked, and then received a practice sheet of six equations. The subjects received error feedback on the practice sheet, but not on the regular sheets. The subjects began working down the list of equations on each sheet when told "go" by the experimenter, who timed the work with a stopwatch calibrated to $.1 \mathrm{sec}$. The subjects were told to go as fast as they could without making any errors. They were told not to return to previously completed equations to check their answers, but that if they detected an error immediately after having made it, to cross it out and enter the correct check mark.

In the addition and subtraction tasks, the answer blanks were $T_{\ldots} \quad F_{\ldots} \ldots$, which represented "true" and "false," respectively. In the oddness tally task, the blanks were 0 2 an even number. In the odd-even choice task, the blanks were ODD _ EVEN__, and the subjects indicated whether the sum had to be odd, for example, $02+13=$ ?, or even, for example, $10+06=$ ? The subjects were told that the sum had to be even if both numbers were odd or both were even, but had to be odd if one number was odd and the other was even.

The subtraction and oddeven choice tasks could both be done using addition. In the subtraction task, the subjects could have checked whether the first term equalled the sum of the second and third terms, as in $17-16=01$. In the odd-even choice task, the subjects could have determined whether the sum of the two terms was odd or even, rather than determining whether both numbers were odd or even. To gauge how frequently subjects used these alternative strategies, they were asked after the session what percentage of the subtraction and odd-even choice equations were done by addition.

Data analysis. To determine the mean RT per equation, the time for the sheet was divided by 20 . Since the time was measured for the sheet as a whole, RT refers to all trials, correct as well as incorrect. Mean RT also could not be distinguished by trial type (true, false), and thus split effects on false trials were attenuated by the inclusion of true trials. Errors on true trials could be distinguished from those on false trials, but because trials were blocked by the size of split, and there might be shifts in response bias between true versus false on particular blocks 
(sheets), the main error analyses were based on total errors per sheet, and that, too, may attenuate split effects.

\section{Results}

The first analysis examined the two arithmetic tasks (addition, subtraction). Mean RT was significantly lower in addition than in subtraction, $1,608 \mathrm{msec}$ versus 1,841 $\operatorname{msec}[\mathrm{F}(1,23)=39.21, \mathrm{p}<.001]$, but errors were not, $2.11 \%$ versus $2.53 \%(\mathrm{~F}<1)$. Direction of split (positive, negative deviation) had no significant effect on either RT $(F<1)$ or errors $(F<1)$. The (absolute) size of split had no significant effect on RT $[F(3,69)=1.81]$, and had only a marginally significant effect on errors $[F(3,69)$ $=2.56, \mathrm{p}<.10]$. As Figure 4 shows, RT and errors generally decreased as size of split increased (symbolic distance factor), but the smoothness of this decrease was disrupted by downward dips in both RT and errors at splits of +1 and +3 , just as predicted by the odd-even rule. Orthogonal components of the split effect were computed to assess the strength of these two tendencies. The linear component, reflecting the linear portion of the symbolic distance effect, was significant on errors $[F(1,69)=4.23, p<.05]$, but not on $\operatorname{RT}[F(1,69)=$ 1.39]. The quadratic component, reflecting curvature or nonlinearity in the symbolic distance effect, was not significant on RT $(F<1)$ or errors $(F<1)$. The cubic component, reflecting the zigzag pattern predicted by the odd-even rule, was marginally significant on both RT $[F(1,69)=3.69, p<.10]$ and errors $[F(1,69)=$ $3.31, \mathrm{p}<.10]$. Pairwise comparisons of adjacent splits showed no effect for a split of \pm 1 versus \pm 2 on RT $(F<1)$ or errors $(F<1)$, or for \pm 3 versus \pm 4 on $R T$

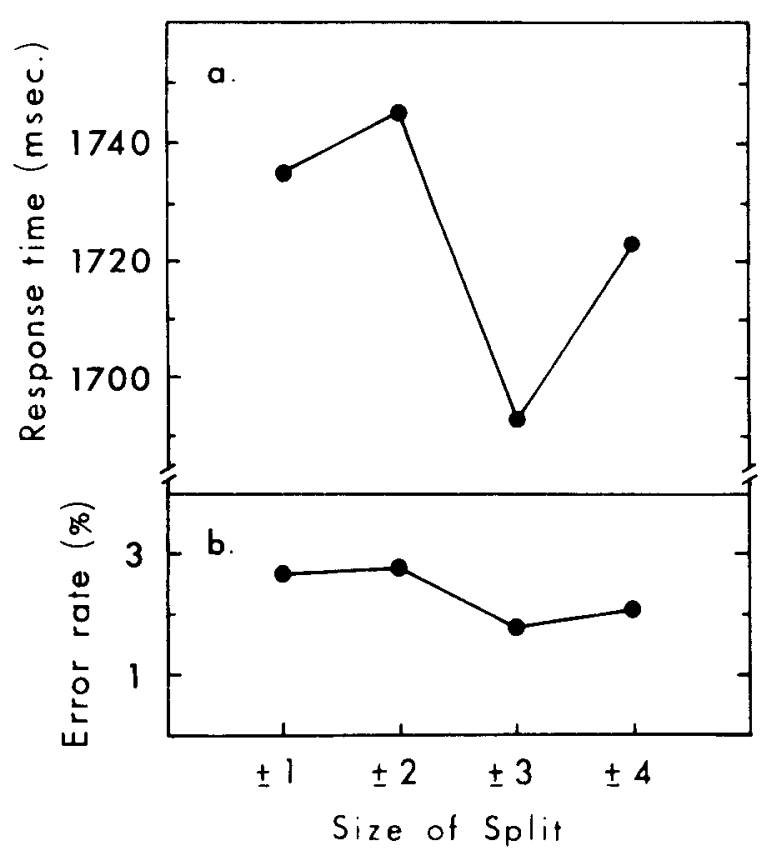

Figure 4. Experiment 2: Mean response time (in milliseconds) and percentage of error rate by (absolute) size of split for sheet (true and false equations).
$[F(1,23)=1.50]$ or errors $(F<1)$, but a significant effect on both RT $[F(1,23)=5.39, p<.05]$ and errors $[F(1,23)=4.55, p<.05]$ between \pm 2 and \pm 3 (see Figure 4).

As Figure 4 shows, a violation of the oddeven rule more than compensated for a decrease of one step in symbolic distance, because both RT and errors were lower on \pm 1 than on \pm 2 and on \pm 3 than on \pm 4 . However, in an additional analysis, performance was not significantly better on odd splits $( \pm 1, \pm 3)$ than on even splits $( \pm 2, \pm 4)$, either on RT $[F(1,23)=1.41]$ or errors $(\mathrm{F}<1)$. In yet a further analysis, we distinguished the 12 "fast odd-even" subjects, who were equally fast or faster on the odd-even choice task than on the addition task, from the 12 "slow odd-even" subjects, who were slower on the odd-even choice task than on the addition task. Type of subject did not have an effect on overall RT or errors (F $<1$ in both cases), but did interact significantly with odd versus even split on errors $[F(1,22)$ $=4.95, \mathrm{p}<.05]$, although not on $\mathrm{RT}[\mathrm{F}(1,22)=1.19]$. On both errors and RT, performance on even splits was as good as or better than that on odd splits for the slow odd-even subjects, but for the fast odd-even subjects, even splits did less well than odd splits.

In a separate analysis of the 12 fast odd-even subjects, performance was significantly better on odd $( \pm 1, \pm 3)$ than on even $( \pm 2, \pm 4)$ splits on errors $[F(1,11)=5.65$, $p<.05]$, and was nearly so on RT $[F(1,11)=3.72$, $\mathrm{p}<.10]$. Errors for these subjects were $2.81 \%, 3.54 \%$, $1.56 \%$, and $2.60 \%$, respectively, for splits of $\pm 1, \pm 2$, \pm 3 , and \pm 4 , whereas RTs were $1,673,1,704,1,625$, and $1,671 \mathrm{msec}$, respectively. For these subjects, size of split had a significant effect on errors $[F(3,33)=4.15$, $\mathrm{p}<.025]$, but not on $\mathrm{RT}[\mathrm{F}(3,33)=1.88]$. The cubic component of this effect, reflecting the zigzag pattem predicted by the odd-even rule, was significant on both errors $[F(1,33)=10.18, p<.01]$ and $R T[F(1,33)=$ $4.90, \mathrm{p}<.05]$. In neither case was the linear or quadratic component significant.

A second analysis classified addition and subtraction errors for all subjects by equation type (true, false). Errors were somewhat, but not significantly, higher on true than on false equations, $2.65 \%$ versus $1.93 \%$ $[F(1,23)=1.11]$. As would be expected, the zigzag pattern in the split function associated with the oddeven rule was more evident on false equations than on true equations. Errors on false equations were $2.38 \%$, $3.15 \%, 0.85 \%$, and $1.33 \%$, respectively, for splits of \pm 1 , $\pm 2, \pm 3$, and \pm 4 . The trial type $x$ split interaction was marginally significant $[F(3,69)=2.45, p<.10]$. Neither the linear component $[F(1,69)=1.69]$ nor the quadratic component $(F<1)$ of this interaction was sig. nificant, whereas the cubic component, reflecting the enhanced zigzag pattern on false equations, was significant $[F(1,69)=5.40, p<.025]$. In a separate analysis of false equations, there was a significant effect of size of split $[F(3,69)=5.15, p<.01]$, for which the linear component $[F(1,69)=7.10, p<.01]$ and the cubic 
component $[F(1,69)=8.23, p<.01]$, but not the quadratic component $(F<1)$, were significant.

A third set of analyses compared each arithmetic task with each odd-even task. The addition task was significantly faster than the oddness tally task, $1,608 \mathrm{msec}$ versus $2,303 \mathrm{msec}[\mathrm{F}(1,23)=62.03, \mathrm{p}<.001]$, and significantly less errorful, $2.11 \%$ versus $5.26 \%[\mathrm{~F}(1,23)=$ $17.78, \mathrm{p}<.001]$. The subtraction task also was significantly faster than the oddness tally task, $1,841 \mathrm{msec}$ versus $2,303 \mathrm{msec}[\mathrm{F}(1,23)=39.11, \mathrm{p}<.001]$, and significantly less errorful, $2.53 \%$ versus $5.26 \%[\mathrm{~F}(1,23)$ $=8.52, \mathrm{p}<.01]$. The addition task did not differ significantly from the odd-even choice task on either RT, $1,608 \mathrm{msec}$ versus $1,633 \mathrm{msec}(\mathrm{F}<1)$, or errors, $2.11 \%$ versus $2.97 \%[F(1,23)=1.54]$. The subtraction task was significantly slower than the odd-even choice task, $1,841 \mathrm{msec}$ versus $1,633 \mathrm{msec}[\mathrm{F}(1,23)=9.70$, $\mathrm{p}<.01]$.

A fourth analysis focused on the oddness tally task. The oddness tally was significantly slower when about half of the equations on the sheet were true than when all were false, $2,392 \mathrm{msec}$ versus $2,214 \mathrm{msec}[\mathrm{F}(1,23)=$ $8.48, \mathrm{p}<.01]$. A fifth analysis classified oddness tally errors by the actual number of odd numbers present. Errors increased significantly as actual number of odd numbers increased $[F(3,69)=6.64, p<.001]$, indicating that misses were more frequent than false-positives. The error rates were $0.34 \%, 4.46 \%, 6.07 \%$, and $8.86 \%$, respectively, when the correct answers were $0,1,2$, and 3.

\section{Discussion}

Experiment 2 confirmed Experiment 1 in finding significant decreases in RT and errors when the (absolute) size of the split increased from \pm 2 to \pm 3 , but not when it increased from \pm 1 to \pm 2 or from \pm 3 to \pm 4 . In fact, RT and errors increased slightly in the latter two cases, indicating that the odd splits of \pm 1 and \pm 3 gained more from violating the odd-even rule than they lost from being smaller than the even splits of \pm 2 and \pm 4 (symbolic distance effect). The odd splits had an advantage over the even splits only among the fast oddeven subjects, who presumably would be most adept at using the odd-even rule to bypass normal addition or subtraction.

The difference between splits of \pm 2 and \pm 3 on RT and errors was about half as large in Experiment 2 (Figure 4) as in Experiment 1 (Figure 3), reflecting the fact that data for true and false trials were combined in Experiment 2. Experiment 2 increased the generality of the odd-even effect, showing it to hold on subtraction or difference verification and when the direction of split was negative. However, it is possible that the oddeven rule was used in the subtraction task only when that task was performed by addition, that is, adding the second term (subtrahend) to the third term (proposed difference) and then comparing that sum to the first term (minuend). Sixteen of the 24 subjects said after the session that they had used addition in the subtraction task, and their reports indicated that addition was used on about $45 \%$ of the subtraction equations.

Four of the 24 subjects (two were fast odd-even subjects, and two were slow) said that they took oddness and evenness into account in the addition task, and one also reported doing so in the subtraction task. One (slow) subject said he picked up the habit early, in the first or second grade. Another (slow) subject said she normally uses the odd-even information if she has enough time to think it out, but that time pressure in the present addition task let her do so on only about $50 \%$ of the trials. As in Experiment 1, most subjects were not aware of using the odd-even rule, which again suggests that the rule was generally applied in an automatic, nonconscious manner.

The good performance obtained on the two odd-even tasks indicates that the subjects were well-versed on the odd-even properties of numbers and could have used this information in an automatic fashion. Performance was nearly as fast and accurate on the odd-even choice task as on the addition task. The present data may overstate the ease of making an odd-even choice, however, because if the task became difficult, the subjects could always resort to addition to determine the answer, rather than check whether both addends were odd or even (even sum) or there was one of each (odd sum). Seventeen of the 24 subjects said that they used addition in the oddeven choice task, and their reports indicated that addition was used on about $40 \%$ of the odd-even choice problems. However, the more important finding is that subjects reported they did not use addition on about $60 \%$ of the trials, presumably because the odd-even rule often could be applied directly in a more rapid fashion, and not simply because they did not think of using addition. The 7 subjects who said they never added on the odd-even choice task were somewhat faster on that task than on the addition task, $1,497 \mathrm{msec}$ versus $1,558 \mathrm{msec}$, although somewhat more errorful, $5.36 \%$ versus $3.04 \%$. Three of the 7 said that the odd-even choice task was the easiest task of the four.

The oddness tally task could not be done using addition, and subjects were about $50 \%$ slower and twice as errorful on it as on addition. However, this task may have been more difficult largely because subjects had to choose among four possible answers $(0,1,2$, or 3 odd numbers) rather than two (true, false). The unfamiliarity of the oddness tally task also may have contributed to its difficulty, and subjects may have had difficulty in ignoring the numerical contents of the equations, because RT was greater when true equations were included.

\section{GENERAL DISCUSSION}

Both experiments confirmed the finding of no difference in performance between splits of \pm 1 and \pm 2 (Hallford, 1982, Experiment 1; Parkman \& Groen, 1971). The lack of an effect here was not due to the 
insensitivity of the present procedure, because splits of \pm 2 and \pm 3 always produced significant differences on both RT and errors. Furthermore, the linear component of the split effect was significant in most cases, indicating that the symbolic distance effect was indeed present. These results favor supplementing the symbolic distance factor with the odd-even rule, which can explain why a split of \pm 1 was generally about as good as a split of \pm 2 and why a split of \pm 3 was always much better than either \pm 1 or \pm 2 . Also favoring the addition of the oddeven rule is the fact that performance was equally good with splits of \pm 3 and \pm 4 . The cubic component of the split effect, reflecting the zigzag pattern found, was significant or marginally significant in most cases. However, the lack of difference between \pm 3 and \pm 4 must be treated with caution, since the symbolic distance effect may not be linear and may simply reach asymptote at or near a split of \pm 4 . The fact that no quadratic component was detected for the split effect in either experiment, however, suggests that the symbolic distance effect was linear over the present range of splits.

Many interesting questions remain on the odd-even rule. At what age do children begin to use it in sum verification? Would explicitly teaching the rule help children to develop greater skill in addition more rapidly? When is the rule applied by adults during processing? The good performance found here on the odd-even tasks indicates that odd-even properties often can be registered quite rapidly, thus allowing the odd-even rule to be applied concurrently with or even prior to the regular addition operation, perhaps as a fast check. The fast odd-even subjects in Experiment 2 were especially likely to benefit when an odd-even violation occurred.

An odd-even violation is just one of many properties or cues that may permit a person to bypass regular processing. Restle (1970) proposed that a fast estimation process, rather than the normal addition operation, is relied upon whenever the proposed sum deviates considerably from the correct sum. Banks, Fujii, and KayraStuart (1976) likewise proposed that in choosing the larger or smaller digit of a pair, a fast decision could be made if the two digits fell in different regions (small, large). Ashcraft and Stazyk (1981) found that RT was lower on false trials having a very large split (13) than on true trials, which "reveals the operation of decision processes and, possibly, an evaluation stage that cooccurs with retrieval" (p. 195). The concurrent evaluation process obtains "global evaluations of the sum of the problem, simply coming up with a progressively more refined region within which the sum is likely to fall" (p. 191). Ashcraft and Stazyk noted that a variety of cues are available for cases such as $14+12=17$; the proposed sum is far too small, and it mismatches the correct sum both in the ones column $(4+2=7)$ and in the tens column $(1+1=1)$. Thus, "other information, drawn from general knowledge about arithmetic, becomes available during the processing of a stimulus and may influence performance on even simple addition tasks" (p. 191).
In multiplication, too, certain properties or cues may permit the bypassing of regular processing on false equations. Thomdike (1922) mentioned two such "protective habits": (1) If a number is multiplied by a number larger than 1 , then the product ought to be larger than the number; (2) if it is multiplied by a number less than 1 , then the product ought to be smaller. With division, the reverse relationships would hold. Duffy and Fisher (1980) found that a false product was rejected $115 \mathrm{msec}$ faster if it were not a multiple of either multiplier, for example, $8 \times 9=41$, than if it were, for example, $8 \times 9=40$. Stazyk, Ashcraft, and Hamann (1982) obtained similar results. The oddeven rule of sum verification can be extended, in modified form, to multiplication. If both multipliers are odd, then the correct product must be odd; else it must be even.

Even when the odd-even properties of the numbers are registered too late to permit the bypassing of regular processing, there still may be a benefit on errors, owing to the check on accuracy. In the present study, in fact, the error effect sometimes was more reliable than the RT effect. Thus, the zigzag pattern (cubic component) of the split effect in Experiment 1 was significant on errors, but not on RT. In terms of practical benefit, the potential improvement in accuracy from use of the oddeven rule may prove far more important than the rather modest improvement to be had in speed.

Whatever makes a proposed sum look obviously incorrect ought to help people to check their arithmetic. Sometimes a false sum may be difficult to reject because the equation is similar to a true equation with a different operation. For example, people find it difficult to reject such equations as $1+7=6$ (Ashcraft \& Battaglia, 1978), for which the answer would be true with subtraction, and $3+3=9$ (Winkelman \& Schmidt, 1974), for which the answer would be true with multiplication. At heart, both odd-even violations and symbolic distance help people by making proposed sums look more obviously incorrect. Thus, it is easier to reject a false proposed sum to the extent that it is dissimilar to the correct sum, owing either to a violation of the odd-even rule or to a large split.

\section{REFERENCES}

Aiken, L. R., \& Williams, E. N. (1973). Response times in adding and multiplying single-digit numbers. Perceptual and Motor Skills, 37, 3-13.

Ashcraft, M. H., \& Battaglia, J. (1978). Cognitive arithmetic: Evidence for retrieval and decision processes in mental addition. Journal of Experimental Psychology: Human Learning and Memory, 4, 527-538.

Ashcraft, M. H., Fierman, B. A., \& Hamann, M. S. (1980, November). The development of network retrieval in addition. Paper presented at the meeting of the Psychonomic Society, St. Louis.

Ashcraft, M. H., \& Hamann, M. S. (1981, November). Children's strategies for solving simple and complex addition problems. Paper presented at the meeting of the Psychonomic Society, Philadelphia.

Ashcraft, M. H., \& STazYK, E. H. (1981). Mental addition: A 
test of three verification models. Memory \& Cognition, 9, 185-196.

Banks, W. P., FujiI, M., \& Kayra-Stuart, F. (1976). Semantic congruity effects in comparative judgments of magnitudes of digits. Journal of Experimental Psychology: Human Perception and Performance, 2, 435-447.

Duffy, S. A., \& Fisher, D. L. (1980, May). The organization and processing of multiplication facts. Paper presented at the meeting of the Midwestern Psychological Association, St. Louis.

Fletcher, B., \& RABbitT, P. M. A. (1978). The changing pattern of perceptual analytic strategies and response selection with practice in a two-choice reaction time task. Quarterly Journal of Experimental Psychology, 30, 417-427.

Gonzalez, E. G., \& Kolers, P. A. (1982). Mental manipulation of arithmetic symbols. Journal of Experimental Psychology: Learning, Memory, and Cognition, 8, 308-319.

Groen, G. J., \& Parkman, J. M. (1972). A chronometric analysis of simple addition. Psychological Review, 79, 329-343.

HALlFoRD, E. W. (1982). Effect of size of numerical difference and stimulus configuration on speed of sum verification. Unpublished master's thesis, Ohio State University, Columbus.

Miller, K., Penlmutter, M., \& Kentina, D. (1984). Cognitive arithmetic: Comparison of operations. Journal of Experimental Psychology: Learning, Memory, and Cognition, 10, 46-60.
Moyer, R. S., \& Landauen, T. K. (1967). Time required for judgments of numerical inequality. Nature, 215, 1519-1520.

Parkman, J. M. (1972). Temporal aspects of simple multiplication and comparison. Journal of Experimental Psychology, 95, 437-444.

Parkman, J. M., \& Groen, G. J. (1971). Temporal aspects of simple addition and comparison. Journal of Experimental Psychology, 89, 335-342.

RESTLE, F. (1970). Speed of adding and comparing numbers. Journal of Experimental Psychology, 83, 274-278.

Russo, J. E., \& Wisher, R. A. (1976). Reprocessing as a recognition cue. Memory \& Cognition, 4, 683-689.

Stazyr, E. H., Ashcraft, M. H., \& Hamann, M. S. (1982). A network approach to mental multiplication. Journal of Experimental Psychology: Learning, Memory, and Cognition, 8, 320-335.

Thonndike, E. L. (1922). The psychology of arithmetic. New York: Macmillan.

Winkelman, J. H., \& Schmidt, J. (1974). Associative confusions in mental arithmetic. Journal of Experimental Psychology, 102, 734-736.

(Manuscript received August 10, 1983;

revision accepted for publication November 29, 1983.) 